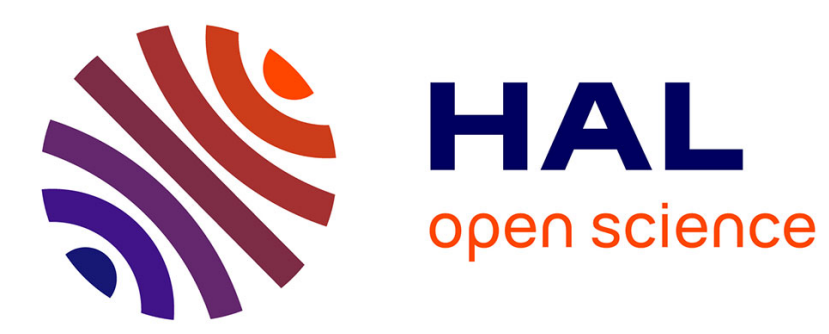

\title{
General Mechanism for the Meandering Instability of Rivulets of Newtonian Fluids
}

\author{
Adrian Daerr, Jens Eggers, Laurent Limat, Nadine Valade
}

\section{To cite this version:}

Adrian Daerr, Jens Eggers, Laurent Limat, Nadine Valade. General Mechanism for the Meandering Instability of Rivulets of Newtonian Fluids. Physical Review Letters, 2011, 106 (18), 10.1103/PhysRevLett.106.184501 . hal-02359232

\section{HAL Id: hal-02359232 https://hal.science/hal-02359232}

Submitted on 16 Nov 2019

HAL is a multi-disciplinary open access archive for the deposit and dissemination of scientific research documents, whether they are published or not. The documents may come from teaching and research institutions in France or abroad, or from public or private research centers.
L'archive ouverte pluridisciplinaire HAL, est destinée au dépôt et à la diffusion de documents scientifiques de niveau recherche, publiés ou non, émanant des établissements d'enseignement et de recherche français ou étrangers, des laboratoires publics ou privés. 


\title{
A general mechanism for the meandering of rivulets
}

\author{
A. Daerr ${ }^{1}$, J. Eggers ${ }^{2}$, L. Limat ${ }^{1}$, and N. Valade ${ }^{1}$ \\ 1 Laboratoire Matière et Systèmes Complexes, UMR 7057 of CNRS and University Paris Diderot, \\ 10 rue Alice Domon et Léonie Duquet, 75025 Paris Cedex 13, France, \\ 2 School of Mathematics, University of Bristol, Bristol BS8 1TW, United Kingdom
}

\begin{abstract}
A rivulet flowing down an inclined plane often does not follow a straight path, but starts to meander spontaneously. Here we show that this instability is the result of two key ingredients: fluid inertia and anisotropy of the friction between rivulet and substrate. Meandering only occurs if the motion normal to the instantaneous flow direction is more difficult than parallel to it. We give a quantitative criterion for the onset of meandering, and confirm it by comparing to the flow of a rivulet between two glass plates which are wetted completely. Above the threshold, the rivulet follows an irregular pattern with a typical wavelength of a few $\mathrm{cm}$.
\end{abstract}

Meandering of rivulets down a vertical or inclined surface is a well known phenomenon [1-10], which is important to understand the degree to which a solid surface is covered by a liquid film: the width of a rivulet is typically $1 \mathrm{~mm}$, while the transversal excursions of a meander may be several $\mathrm{cm}$. The small-scale meanders discussed in this paper also fall into a broader class of instabilities which include river meandering [11] and the coiling of fluid jets [12]. Most explanations of meandering are based on the idea that centrifugal forces amplify the curved parts of the stream $[1,4,8]$, while others emphasize the role of noise and surface disorder $[9,10]$. However, as yet there exists no quantitative theory for the meandering of rivulets.

Most available experiments were performed in partial wetting conditions, and on relatively rough substrates, which suffer from contact angle hysteresis, and the pinning of the rivulet on defects. Thus the mechanism of instability remains hidden by noise, and quantitative comparison is difficult. Instead, we present meandering experiments in a situation of complete wetting, where hysteresis and surface defects do not matter. To this end we confined a fluid with low surface tension (e.g. silicone oil) between two parallel plates (the Hele-Shaw geometry), so a rivulet forms as a long liquid bridge or film connecting the two plates, see Fig. 1. The insides of the glass plates are covered with a thin fluid film, since the silicone oil strongly favors wetting. Previous meandering experiments in the same geometry have only been reported with a surfactant aiding the stability of the bridge $[3,13]$. This makes the results difficult to reproduce, and poses questions as to the role of complex surfactant rheology.

In this letter, we show that a meandering instability exists for several inert, Newtonian fluids between two glass plates, thus establishing its universal nature. By varying the flow rate, we show that the rivulet meanders above a well-defined threshold. Based on a hydrodynamic description, we derive an explicit and parameter-free criterion for the threshold value of the critical flow speed above which meandering occurs. We observe that the one crucial ingredient necessary for meandering is that it is harder for the fluid to move in a direction perpendicu-

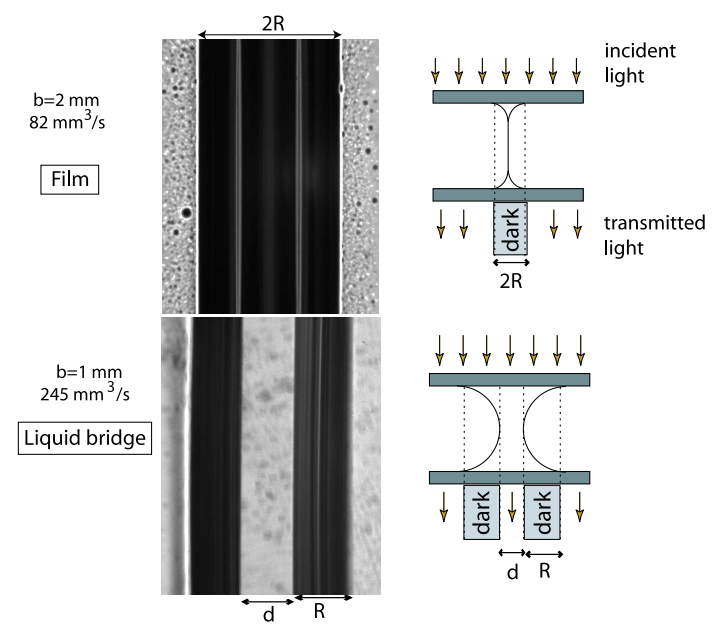

FIG. 1. Silicone oil forms a rivulet between two glass plates, whose cross section can either be a film (top) or a liquid bridge (bottom). Dimensions are $R=0.7 \mathrm{~mm}$ at the top, and $R=0.5 \mathrm{~mm}$ at the bottom. On the left, closeup frontal views of the glass plates, used to measure the rivulet width. On the right, a schematic of the rivulet cross-section. At low flow rates and large plate separations $b$, a film forms (top); the rivulet is imaged as a single black line, and the cross-sectional area is $A=(4-\pi) R^{2}$. At higher flow rates or small separation, a liquid bridge appears (bottom), imaged as a bright line bounded by two black lines. The area is $A=(4-\pi) R^{2}+b d$.

lar to the instantaneous direction of the rivulet, than it is to move in a downstream direction. An analogy would be that of a car that can only overturn if the tires have sufficient traction.

Our experiment consists of a Hele-Shaw cell made of two glass plates of dimensions $100 \mathrm{~cm} \times 30 \mathrm{~cm}$ and thickness $5 \mathrm{~mm}$, arranged at an angle $\alpha$ relative to the horizontal. The plate spacing $b$ varies between 0.6 and $2 \mathrm{~mm}$. A gear pump injects fluids of viscosity between 0.9 and 3 times that of water at the top of a cell through a hypodermic needle; typical flow rates lie between 50 and 1000 $\mathrm{mm}^{3} / \mathrm{s}$. The shape assumed by the rivulet in the plane of the cell is followed by video recording, the cell being lit by Neon tubes on either side. Closeup views (see Fig. 1) 


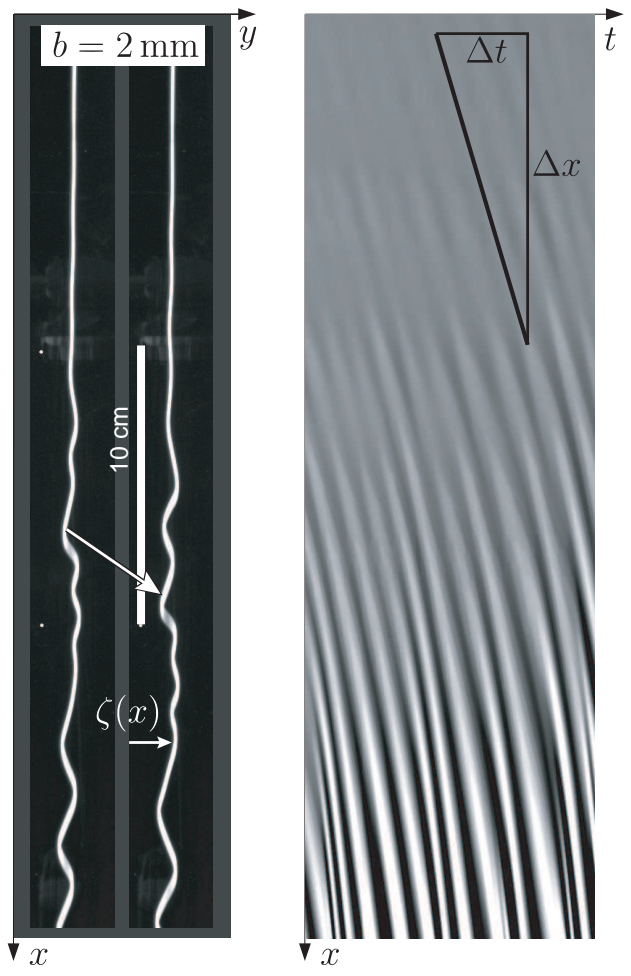

FIG. 2. Left: Two successive images of meandering patterns (system A, $Q=182 \mathrm{~mm}^{3} / \mathrm{s}$ ), with $\Delta t=0.33 \mathrm{~s}$ between them. The spatial shift, indicated by an arrow, shows the phase speed $u_{\phi}=2.42 \mathrm{~cm} / \mathrm{s}$. Note the irregularity of the pattern. Right: a spatiotemporal plot (same spatial scale, grey value proportional to $\zeta(x, t))$; the triangle indicates the measurement of $u_{\phi}$ in the linear regime.

with a light source far behind the plates are used to measure the width of the rivulet and thus its cross section. Depending on the flow rate relative to plate spacing, two different states are observed. At low flow rates, a thin liquid film, connected to the plate through Plateau borders, which shows up as a single dark line. At higher flow rates, a liquid bridge, which appears as a bright line with dark borders (cf. Fig. 1). In Figs. 2 and 3, detailed results are given for system A, which is specified in Table I. Results for other parameter values are also given in Table I.

At low flow rates, the rivulet remains perfectly straight over the whole length of the Hele Shaw cell. Moreover, its width stays constant over the whole length of the cell, indicating that the flow is fully developed from near the inlet, and the mean flow speed is determined by a balance of gravity and viscous friction. Above a critical flow rate meandering patterns begin to develop, which are irregular in general, and which are convected downstream, as shown in Fig.2. Typical wavelengths are of the order of a few $\mathrm{cm}$, much larger that the rivulet width of a few $\mathrm{mm}$. The phase speed $u_{\phi}$ of the meandering pattern is illustrated in Fig. 2.
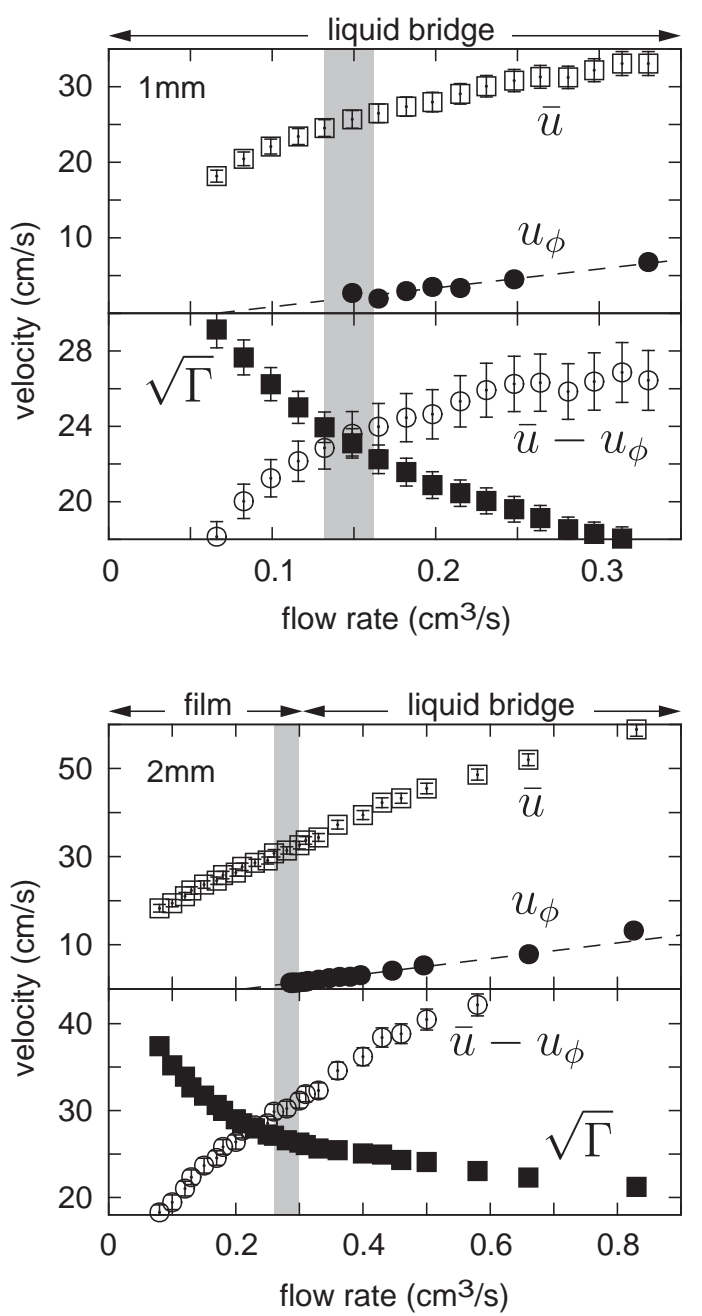

FIG. 3. Experimental data for system A, $b=1 \mathrm{~mm}$ (top) and $b=2 \mathrm{~mm}$ (bottom). The top graph shows the mean velocity $\bar{u}(\square)$ and phase velocity $u_{\phi}(\bullet)$ as function of flow rate $Q$. The bottom graph shows the surface tension parameter $\sqrt{\Gamma}$ (ם) and velocity difference $\Delta u=\bar{u}-u_{\phi}$ (०), where $u_{\phi}$ is determined from a linear approximation to the data, dashed line; we put $u_{\phi}=0$ if the linear approximation falls below zero. Theory predicts a transition at the crossing. The shaded region marks the observed threshold to within the error.

In Fig. 3, on the top of each graph we show the mean velocity $\bar{u}$ of the flow inside the rivulet, and the phase speed $u_{\phi}$ of the meandering pattern, for two different plate spacings. We do not measure $u_{\phi}$ below the transition, since it would require us to excite perturbations externally. Instead, we extrapolate the data linearly to below the transition. The mean velocity is calculated from $\bar{u}=Q / A$, where the cross-sectional area $A$ is calculated according to the diagrams in Fig. 1 (see caption); in general, $A$ will depend on $Q$. In the film regime, we measure the width $2 R$ of the dark line, where $R$ is the radius of curvature of the Plateau border, and $b-2 R$ the length of the film. In the liquid bridge regime we mea- 
sure the width $d$ of the bright line as well as the width $R$ of the dark borders, which is now the radius of curvature of the meniscus bordering the bridge. All surfaces are assumed to be arcs of circles, with contact angle $0^{\circ}$. The phase speed $u_{\phi}$ is determined from a linear fit to the spatio-temporal plot of the meandering pattern (cf. Fig. 2).

To explain the observed instability theoretically, we use the two-dimensional Navier-Stokes equation, as obtained from averaging the flow in the $z$-direction (perpendicular to the plates), as customary for Hele-Shaw flow [14]. The velocity field is $\mathbf{u}=(u, v)$, whose components correspond to the coordinate directions $x, y$, as shown in Fig.2, and $\mathbf{n}$ is the normal to the rivulet path $\zeta(x)$ :

$$
\partial_{t} \mathbf{u}+\beta(\mathbf{u} \cdot \nabla) \mathbf{u}=-k \mathbf{u}+\left(\Gamma \kappa-k_{c l} u_{n}\right) \mathbf{n}+g \mathbf{e}_{x} .
$$

Here $\Gamma$ is the effective line tension of the rivulet, and $k_{c l}$ the additional "contact line" friction associated with the rivulet possessing a nonzero velocity $u_{n} \equiv \mathbf{u} \cdot \mathbf{n}$ normal to itself. For Poiseuille flow the friction factor is $k=12 \nu / b^{2}$ ( $\nu$ the kinematic viscosity) and $\beta=6 / 5$. Since the viscosity is small, we expect our profile to be closer to a plug flow and thus $\beta$ to be close to unity $[14,15]$. In the absence of a more precise estimate, and in the interest of simplicity, we set $\beta=1$ in the following. The contact line friction comes from the fact that normal motion causes a shear flow in the junction between the thin film of thickness $h$ covering the plates, and the rivulet. This is analogous to the Landau-Levich problem [16] viewed in a frame of reference moving with the plate which drags out the film. Estimating the dissipation in the junction, one finds [17] that $k_{c l} \propto k \sqrt{b / h}$.

A second contribution to the normal force comes from the curvature $\kappa$ of the rivulet, since a curved rivulet has a greater surface area. In the case of a liquid bridge of finite width (cf. Fig. 1 (bottom)), it was shown in [18] that the pressure difference across the meniscus in the small-speed limit is $\Delta p=-\gamma \pi \kappa / 4$. Thus $\Gamma=2 \Delta p b /(\rho A)$ in (1), since the acceleration is proportional to the total force $2 \Delta p \ell b$ per length $\ell$ of the rivulet (accounting for the 2 sides of the rivulet), divided by the mass $\rho \ell A$. Here $\gamma$ the surface tension, and $\rho$ the density of the fluid. In the film regime the interface is planar over a length $b-2 R$, which contributes a factor $\Gamma=2 \gamma(b-2 R) /(\rho A)$, the other part is curved with a radius of curvature $R$ (cf. Fig. 1 (top)). A combination of the contribution of both curved and straight interfaces gives the generalized formula $\Gamma=$ $\gamma(2(b-2 R)+R \pi) /(\rho A)$.

We now perform a linear stability analysis of (1), assuming small deviations of the rivulet position $\zeta(x)$ from the vertical (cf. Fig. 2). For $u$, we can take the mean velocity $\bar{u}=g / k$, so the convection of the rivulet is described by

$$
\zeta_{t}+\bar{u} \zeta_{x}=v
$$

and the $y$-component of the Navier-Stokes equation (1) is

$$
v_{t}+\bar{u} v_{x}=-k v-k_{c l}\left(v-\bar{u} \zeta_{x}\right)+\Gamma \zeta_{x x},
$$

with subscripts denoting partial differentiation. In a linear approximation, the normal velocity of the rivulet is related to the velocity $(\bar{u}, v)$ relative to the plate by $u_{n}=v-\bar{u} \zeta_{x}$. Using (2), the variable $v$ can be eliminated from (3), leading to

$$
\zeta_{t t}+2 \bar{u} \zeta_{x t}+\bar{u}^{2} \zeta_{x x}=-\left(k+k_{c l}\right) \zeta_{t}-k \bar{u} \zeta_{x}+\Gamma \zeta_{x x} .
$$

Note that the $\bar{u}^{2}$-term stands for centrifugal forces such as those invoked in $[1,2,8,13]$, but inertia by itself does not lead to instability. To illustrate this, let us consider an ideal case in which the right hand side of (4) is negligible. If one seeks solutions of the form $\zeta(x, t) \propto \exp (i q x+\sigma t)$, the dispersion relation has the solution $\sigma=-i q \bar{u}$, i.e. there is only propagation without amplification. If one includes frictional terms proportional to $k$ as well as surface tension forces $\Gamma$, this only leads to damping of an initially excited wave.

However, with contact line friction $k_{c l}$ the full dispersion relation for (4) reads

$$
\sigma=-i q \bar{u}-\bar{k} \pm \sqrt{-\Gamma q^{2}+\bar{k}^{2}+i k_{c l} \bar{u} q},
$$

where $\bar{k}=\left(k+k_{c l}\right) / 2$. It is confirmed easily that the second branch is always damped, while the first becomes unstable if

$$
k_{c l}^{2} \bar{u}^{2}>4 \bar{k}^{2} \Gamma
$$

i.e. when centrifugal forces overwhelm surface tension. The friction term $k_{c l}$ singles out the momentary shape of the rivulet as a preferred path, and thus acts as a constraint, producing a force of constraint (i.e. a centrifugal force), and leading to instability.

This idea can be expressed more formally by observing that at the threshold, (5) becomes $\sigma=-i \bar{u} q k /(2 \bar{k})$, thus the meandering pattern moves with a phase speed

$$
u_{\phi}=\frac{k}{2 \bar{k}} \bar{u} .
$$

This permits to put the stability criterion (6) into a parameter-free form:

$$
\left(\bar{u}-u_{\phi}\right)^{2}>\Gamma,
$$

which is the main result of this paper. According to (8), centrifugal forces on the rivulet arise from the speed $\Delta u=\bar{u}-u_{\phi}$ of a fluid particle in the frame of reference of the pattern in which it moves. Note that the substantial difference between $\bar{u}$ and $u_{\phi}$, evident in Fig. 3, results from the fact that the inclined parts of the stream are slowing down the motion of the meandering pattern, 


\begin{tabular}{cccccccccc}
\hline \hline & $\begin{array}{c}\mathrm{b} \\
(\mathrm{mm})\end{array}$ & $\begin{array}{c}\nu \\
(\mathrm{cP})\end{array}$ & $\begin{array}{c}\rho \\
\left(\mathrm{g} / \mathrm{cm}^{3}\right)\end{array}$ & $\begin{array}{c}g \sin \alpha \\
\left(\mathrm{m} / \mathrm{s}^{2}\right)\end{array}$ & $\begin{array}{c}\gamma \\
(\mathrm{mN} / \mathrm{m})\end{array}$ & $\begin{array}{c}\bar{u} \\
(\mathrm{~cm} / \mathrm{s})\end{array}$ & $\begin{array}{c}u_{\Phi} \\
(\mathrm{cm} / \mathrm{s})\end{array}$ & $\begin{array}{c}\bar{u}-u_{\Phi} \\
(\mathrm{cm} / \mathrm{s})\end{array}$ & $\begin{array}{c}\sqrt{\Gamma} \\
(\mathrm{cm} / \mathrm{s})\end{array}$ \\
\hline $\mathrm{A}$ & 2 & 3 & 0.9 & 9.81 & 17.7 & $31.4 \pm 0.8$ & $1.2 \pm 0.1$ & $30.2 \pm 0.9$ & $26.6 \pm 0.5$ \\
$\mathrm{~A}$ & 1 & 3 & 0.9 & 9.81 & 17.7 & $25.7 \pm 1.2$ & $2.1 \pm 0.1$ & $23.6 \pm 1.2$ \\
$\mathrm{~B} 1$ & 1 & 2 & 1.75 & 8.50 & 14 & $12.1 \pm 0.5$ & $1.1 \pm 0.2$ & $11.0 \pm 0.6$ & $12.3 \pm 1.1$ \\
$\mathrm{~B} 2$ & 0.6 & 0.9 & 1.75 & 4.91 & 13.6 & $17.6 \pm 1.6$ & $0.4 \pm 0.1$ & $17.2 \pm 1.6$ & $21.0 \pm 2.3$ \\
$\mathrm{C}$ & 3.0 & 1.0 & 1.04 & 9.81 & 0 & $0.027 \pm 0.009$ & $0.027 \pm 0.006$ & $0.0 \pm 0.011$ \\
$\mathrm{C}$ & $(3 \mathrm{D})$ & 1.0 & 1.04 & 9.81 & 0 & 0.1 & 0.1 & 0.0 \\
\hline \hline
\end{tabular}

TABLE I. Systems: [A] PDMS oil in air, [B1] perfluorinated oil in air, [B2] perfluorinated oil in air, $[\mathrm{C}] 1 \mathrm{M} \mathrm{NaCl}$ solution in water, in a Hele-Shaw cell [19], and in 3D [12]. According to (8), the last two columns should agree.

because of the extra resistance to normal motion of the contact line, a fact expressed mathematically by (7).

All parameters appearing in (8) are experimentally accessible, so we can test our theory quantitatively, and without adjustable parameters. In Fig. 3 (bottom graph), we have plotted $\Delta u$ and $\sqrt{\Gamma}$, which according to (8) should cross at the transition; the meandering transition observed by us experimentally is marked by the shaded region, which indeed coincides well with the crossing of the two curves. This is confirmed by all the threshold data reported in Table I, for three different fluid systems, and for a considerable range of plate spacings. In particular, we included data from an earlier work with two miscible fluids $[12,19]$ (zero surface tension). In that case, the transition occurs when $u=u_{\phi}$, a finding in agreement with (8), but previously unexplained. The theory also agrees in spirit with the opposite extreme of pinned contact lines [8], for which $u_{\phi}=0$, However, pinning forces have to be included to explain the observed threshold quantitatively [8].

However, one aspect we do not yet understand is the selection of a typical wavelength, as illustrated in Fig. 2. Above the threshold, (5) predicts all wavelengths to turn unstable at the same time, since there is no maximum in the dispersion relation which would select a most unstable wavelength. One physical effect we have not yet taken into account is the regularization at short wavelengths, which are of the order of the width of the rivulet. However, this will not explain the observed wavelengths, which are much larger than the rivulet width. Rather, we suspect that selection occurs on account of a nonlinear mechanism. This is consistent with the irregular appearance of meanders, whose shape is quite far from sinusoidal, and which exhibit a very considerable spread in wavelength.

In conclusion, we have presented a theory for the meandering of rivulets, which agrees quantitatively with experiment. While large-scale meandering, for example of rivers, is governed by very different mechanisms, we be- lieve nonetheless that the basic physical principle behind the instability is the same as the one described by us. On one hand, the driving force ultimately comes from the inertia of the fluid. On the other hand, for this force to act one needs a reference frame, which in the case of rivers is furnished by the existence of a river bed.

[1] J. B. Culkin and S. H. Davis, AIChE J. 30, 263 (1984).

[2] T. Nakagawa and J. C. Scott, J. Fluid. Mech. 149, 89 (1984).

[3] A. Anand and A. Bejan, J. Fluids Eng. 108, 269 (1986).

[4] R. Bruinsma, J. Phys. (France) 51, 829 (1990).

[5] P. Schmuki and M. Laso, J. Fluid Mech. 215, 125 (1990).

[6] T. Nakagawa, J. Multiphase Flow 18, 455 (1992).

[7] H. Kim, J. Kim, and B. Kang, J. Fluid. Mech. 498, 245 (2004).

[8] N. Le Grand, A. Daerr, and L. Limat, Phys. Rev. Lett. 96, 254503 (2006).

[9] B. Birnir, K. Mertens, V. Putkaradze, and P. Vorobieff, J. Fluid Mech. 607, 401 (2008).

[10] B. Birnir, K. Mertens, V. Putkaradze, and P. Vorobieff, Phys. Rev. Lett. 101, 114501 (2008).

[11] L. B. Leopold and M. G. Wolman, Geol. Soc. Am. Bull. 71, 769 (1960).

[12] C. Dombrowski, B. Lewellyn, A. I. Pesci, J. M. Restrepo, J. O. Kessler, and R. E. Goldstein, Phys. Rev. Lett 95, 184501 (2005).

[13] W. Drenkhan, S. Gatz, and D. Weaire, Phys. Fluids 16, 3115 (2004).

[14] F. Plouraboué and E. J. Hinch, Phys. Fluids 14, 922 (2002).

[15] C. Ruyer-Quil, C. R. Acad. Sci., Ser. IIb: Mec., Phys., Chim., Astron. 329, 1 (2001).

[16] L. D. Landau and B. V. Levich, Acta physico-chimica USSR 17, 42 (1942).

[17] J. Eggers, unpublished(2008).

[18] C.-W. Park and G. M. Homsy, J. Fluid Mech. 139, 291 (1984).

[19] A. I. Pesci, M. A. Porter, and R. E. Goldstein, Phys. Rev. E 68, 056305 (2003). 